\title{
Interpolation-Free Coregistration and Phase-Correction of Airborne SAR Interferograms
}

\author{
Pau Prats, Student Member, IEEE, Andreas Reigber, Member, IEEE, and Jordi J. Mallorqui, Member, IEEE
}

\begin{abstract}
This letter discusses the detection and correction of residual motion errors that appear in airborne synthetic aperture radar (SAR) interferograms due to the lack of precision in the navigation system. As it is shown, the effect of this lack of precision is twofold: azimuth registration errors and phase azimuth undulations. Up to now, the correction of the former was carried out by estimating the registration error and interpolating, while the latter was based on the estimation of the phase azimuth undulations to compensate the phase of the computed interferogram. In this letter, a new correction method is proposed, which avoids the interpolation step and corrects at the same time the azimuth phase undulations. Additionally, the spectral diversity technique, used to estimate registration errors, is critically analyzed. Airborne L-band repeat-pass interferometric data of the German Aerospace Center (DLR) experimental airborne SAR is used to validate the method.
\end{abstract}

Index Terms-Calibration, image registration, interferometry, motion compensation, repeat-pass interferometry, synthetic aperture radar (SAR).

\section{INTRODUCTION}

A IRBORNE synthetic aperture radar (SAR) systems usually record the platform movement to later carry out motion compensation during data processing. Due to the lack of accuracy in the navigation system, residual motion errors appear in the image. The most important effects of these residual motion errors are a displacement of the maximum of the impulse response in azimuth, as well as a phase offset. Therefore, the generated interferogram will show registration and interferometric phase errors, in principle corresponding to the occurred baseline error. Both effects mainly take place along the azimuth dimension and are almost constant along range in a beam-center geometry. Residual motion error effects are mostly noticeable in repeat-pass systems, but the solution to be presented can also be applied to single-pass systems.

Regarding the correction of residual motion errors in interferometry, mainly two techniques are described in literature [1],

Manuscript received December 11, 2003; revised March 13, 2004. This work was supported by the Spanish MCYT and FEDER funds under project TIC 2002-04451-C02-01 and by the Catalan Commission for Research (CIRIT).

P. Prats is with the Department of Signal Theory and Communications, Universitat Politècnica de Catalunya (UPC), Barcelona E-08034, Spain, and also with the Department of Telecommunications and Engineering Systems, Universitat Autònoma de Barcelona (UAB), Bellaterra E-08193, Spain (e-mail: pprats@tsc.upc.es).

A. Reigber is with the Department of Computer Vision and Remote Sensing, Technical University Berlin, Berlin 10623, Germany (e-mail: anderl@fpk.tuberlin.de).

J. J. Mallorqui is with the Department of Signal Theory and Communications, Universitat Politècnica de Catalunya (UPC), Barcelona E-08034, Spain (e-mail: mallorqui@tsc.upc.es).

Digital Object Identifier 10.1109/LGRS.2004.828181
[2]. In this letter, both techniques are presented and compared. Additionally, the spectral diversity technique (i.e., splitting of the spectra into subapertures) is analyzed, highlighting the potentials and limitations of using it to measure registration errors [3] as well as residual motion errors. This includes in particular a detailed look on how to adjust in an optimum way the parameters chosen for this approach, when quick nonlinear variations of the residual motion errors are involved. It is important to state that these techniques estimate the differential error between both acquisitions, and not the individual error of each image.

The second issue of this letter is the optimal correction of residual motion errors. Up to now, both effects were taken into account separately during SAR processing. First, a phase correction was applied to the interferogram or to one of the images to correct the azimuth phase undulations, and afterward the registration error was corrected by means of an interpolation. However, noticing the same origin of both effects, a single-step approach is possible. In Section II-C, a novel correction technique is proposed, which removes both the registration errors and the residual phase errors without the use of interpolations, but by refocusing with a modified motion compensation function.

\section{RESIDUAL MOTION ERRORS}

For the following, it is important to state first that in airborne SAR interferometry, there are azimuth registration errors and azimuth phase undulations because of residual motion errors. Of course, master and slave images have usually been processed in order to have the same azimuth pixel spacing, and errors due to the assumption of constant height during motion compensation are considered small compared to residual motion errors. These errors can be minimized with the use of an external digital elevation model (DEM) [4], like the ones provided by the Shuttle Radar Topography Mission mission, now available to the public.

The consequences of unmeasured motion of the platform in an image are well stated in the literature: a constant error induces a phase offset; a linear error induces a shift of the impulse response; and a quadratic one induces defocusing [5]. Therefore, mean phase errors along the synthetic aperture will give interferometric phase offsets (phase undulations), and linear components will result in the displacement of the impulse response.

\section{A. Estimation of Residual Motion Errors Measuring the Registration Error}

In [1], a technique able to detect residual motion errors was presented. It was based on the measurement of the registration error, as this is one of its two main effects. After integration of azimuth displacements, an estimation of residual motion errors 
can be obtained. Then, the interferogram, or one of the images, can be phase-corrected using this information.

\section{B. Direct Estimation of Residual Motion Errors With Spectral Diversity}

The second approach described in the literature [2], which estimates the true residual motion error, uses the spectral diversity technique (i.e., splitting of the spectra, and in this case, the azimuth spectra), either by using two interferograms processed with different squints or by splitting the spectra of both images to generate two low-resolution interferograms. The interpretation of what is seen when generating the differential interferogram is the following: a different squint means targets are focused using different parts of the track. Therefore, the observed phase error is different in the presence of residual motion errors, leading to a nonzero differential phase. This differential phase is indeed the derivative of the residual motion errors (note that the order in which subapertures are multiplied when generating the differential interferogram only changes the sign of the result, which must be taken into account when computing the correction).

The azimuth distance between the center of both subapertures is a key parameter in order to be able to track fast variations of residual motion errors. It is given by

$$
\Delta x(r)=\left(\tan \left(\beta_{1}\right)-\tan \left(\beta_{2}\right)\right) \cdot r
$$

where $\beta_{1}$ and $\beta_{2}$ are the squint centers of each subaperture, and $r$ is the range distance. Distance $\Delta x$ has to be small enough to track fast variations of residual motion errors.

The second important key parameter is the bandwidth of the subapertures. As already commented, the undulations visible in the interferogram are the result of the averaging of the true residual motion error along the synthetic aperture: a long aperture cancels out frequency components much higher than its own bandwidth. Consequently, the larger the subaperture, the less accurate the estimation of the residual motion error gets at a given along-track position. This fact also becomes important when applying the correction. If a small bandwidth is used for the subapertures, then fast variations of residual motion errors are detected. However, phase undulations present in the interferogram might be not so fast due to the aforementioned averaging. Therefore, the correction of phase undulations by directly multiplying the phase correction to the interferogram, as suggested in [2], might lead to overcompensation. The solution presented in Section II-C circumvents this problem.

In order to reduce noise in the differential interferogram, the separation and the bandwidth should be large, as commented in [2] and [3]. Obviously, this imposes a compromise between accuracy in the measurement of derivatives of residual motion errors and noise reduction. In any case, a multilook window in both azimuth and range dimensions is applied, allowing to keep the separation and the bandwidth small. The averaging is needed, as the differential phase is very noisy [2], [3].

To estimate residual motion errors, the differential phase has to be integrated [2]

$$
\tilde{\phi}_{\mathrm{err}}(x, r)=\int_{0}^{x} \frac{\phi_{\mathrm{dif}}\left(x^{\prime}, r\right)}{\Delta x(r)} d x^{\prime}+C
$$

where $\tilde{\phi}_{\text {err }}$ is the estimated residual motion error (in radians), $\phi_{\text {dif }}$ is the phase of the differential interferogram, and $C$ and integration constant. Therefore, the baseline error in line of sight (LOS) is

$$
\Delta r(x, r)=\frac{\lambda}{4 \pi} \tilde{\phi}_{\mathrm{err}}(x, r) .
$$

It has to be noted that when applying any kind of spectral diversity technique, special care must be taken concerning high squint angles, due to the coupling between azimuth and range signals [6].

\section{Residual Motion Compensation}

Once residual motion errors have been estimated, the correction phase has the following expression (it is assumed that the differential interferogram has been generated by multiplying the low-frequency subaperture interferogram by the complex conjugate of the high-frequency subaperture interferogram):

$$
\phi_{\text {corr }}(x, r)=-\frac{4 \pi}{\lambda} \Delta r(x, r)
$$

with $\Delta r$ being the error in meters in LOS as defined in (3). Up to now, in order to correct the effects of residual motion errors, the derived correction phase $\phi_{\text {corr }}$ was usually multiplied to one of the images, followed by a resampling in order to correct the misregistration. However, a better approach is possible. As both effects of residual motion errors (misregistration and azimuth phase undulations) have the same origin, they can also be corrected in one step. Once the differential motion error is known, one can also correct for the phase errors by modifying accordingly the matched filter function used for azimuthal SAR focusing. Such a step can be deemed as a residual motion compensation step, coming after the conventional motion compensation based on navigation data. This approach has the advantage that both the misregistration and the phase errors are corrected in one operation. Therefore, a time-consuming and imprecise interpolation of the data is not necessary.

Such a residual motion compensation step requires decompression of the data along the azimuth dimension (as the assumption that the data are already focused is made, so there is no need to reprocess them again), complex multiplication of the data with (4) in time domain, and subsequently again a compression of the data. Note that in range-Doppler and chirp scaling algorithms, the decompression function would be the complex conjugate of the one used to compress the signal [8]

$$
H_{\text {decomp }}\left(f_{a} ; r_{0}\right)=\exp \left[j \frac{4 \pi}{\lambda} r_{0}\left(1-\sqrt{1-\frac{\lambda \cdot f_{a}}{2 \cdot v}}\right)^{2}\right]
$$

where $f_{a}$ is the azimuth frequency, $\lambda$ the wavelength, $v$ the forward velocity of the platform, and $r_{0}$ the slant range distance. Decompression must be carried out for each range, so that the length of the impulse response after azimuth decompression precisely corresponds to the one of the raw data. Special care must be taken with algorithms like $\omega-k$, where the compression filter is tuned to a single range [7].

This approach is more accurate than interpolation, but has the computational burden of four additional fast Fourier transforms along the azimuth. However, it was found that when using modern fast Fourier transform implementations, the proposed approach can even be faster than the interpolation/phase multiplication approach. The correction can be applied either on one image, or alternatively fractions of it on both images. In both cases, some errors are corrected, but at the same time others induced. However, it has to be noted that the induced motion errors 
are of the same magnitude as the existing ones. The key point is that differential errors are corrected, i.e., residual motion errors are the same in both images. Consequently, the interferogram will appear with no registration errors nor azimuth phase undulations. The most accurate approach is to apply $50 \%$ of the correction to each image, as it is unknown how much error corresponds to each of them. In any case, an improvement in the focusing quality cannot be expected, as the two individual motion errors remain unknown.

It is assumed that the errors result mainly from vertical and horizontal displacements and not from errors in the measurement of the forward velocity. This approximation is quite accurate, as for the same effect on the misregistration, a much stronger velocity error is necessary.

\section{Practical Considerations}

Some differences can be pointed out between the techniques presented in Sections II-A and B. The former computes the correction from the registration error, while the latter uses the differential phase between subapertures. This implies that given a good estimation of the registration error is used, Section II-A estimates the phase undulations appearing in the interferogram, and not the true residual motion error. That is to say, it estimates a smoothed version of residual motion errors. Only when the error is linear along the synthetic aperture, Sections II-A and B result in the same estimated residual motion error. Therefore, the second technique is better suited to detect fast variations (with the appropriate subaperture configuration) than the first technique, thus obtaining a better estimation of residual motion errors. When applying the correction technique presented in Section II-C, the usage of Section II-B will result in a better performance of the overall correction process.

From the application point of view, both methods are equally valid in the sense of getting rid of phase undulations. However, in case of Section II-A, the obtained interferogram might still not have the maximum possible coherence. The true residual motion error is not corrected here, and phase artifacts due to higher order terms, which are not common to both images, do not cancel during interferogram generation.

\section{E. Discussion on the Estimation of Registration Errors With Spectral Diversity}

The suggested technique in [1] to measure the registration error was based on spectral diversity [3]. The assumption made in [3], that the registration error does not change between different subapertures, might not be true in the presence of residual motion errors. As already commented, the displacement of the impulse response is related to the linear component of the motion error along the synthetic aperture. Only as long as the error is exactly linear along the synthetic aperture, the spectral diversity technique results in a correct estimation of the registration error. Of course, several parameters must be taken into account, most importantly the processing bandwidth, which specifies the length of the synthetic aperture, and the accuracy of the navigation system.

In practice, the shorter the synthetic aperture gets, the more probable the residual error is linear along it. This fact has to be considered when choosing the appropriate bandwidth for spectral diversity coregistration. For example, in case of L-band airborne experimental SAR (E-SAR) data, it was found that by pro-

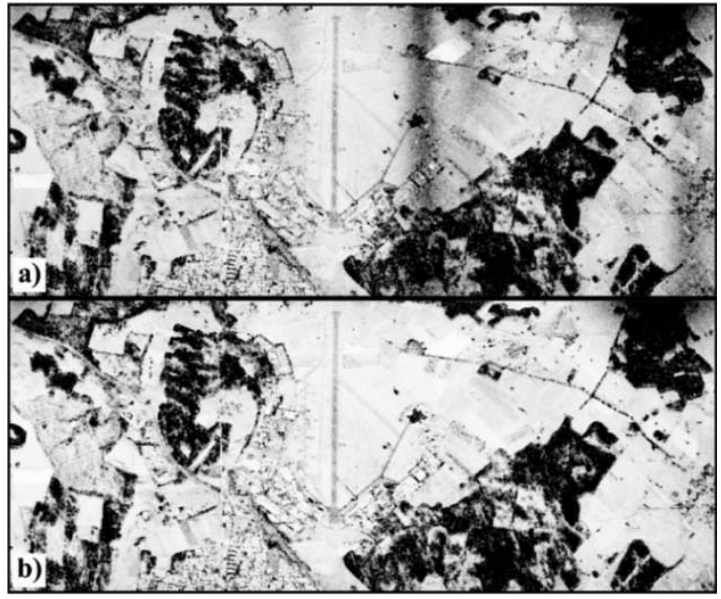

Fig. 1. Coherence (a) before and (b) after applying the proposed correction method.

cessing only one quarter of the full pulse repetition frequency (PRF), residual errors can be considered almost linear along the synthetic aperture. In such a case, spectral diversity can be expected to give a good estimation of the registration errors along azimuth. Certainly, this value is not general, as the characteristics of residual motion errors depend on the navigation system and on the conditions of the data acquisition. In any case, the most precise estimation of the registration error can be obtained using the maximum separation between subapertures, as the linear trend that induces registration errors is better estimated.

It is important to note that when Section II-A uses [3], it is very closely related to Section II-B. However, due to the different interpretations given to spectral diversity differential phase, results might not be exactly the same, as already pointed out in Section II-D.

\section{EXPERIMENTAL RESULTS}

To validate the proposed method, with any loss of generality, airborne repeat-pass E-SAR data were used. The measurements were made at L-band $(1.3 \mathrm{GHz})$ with a bandwidth of $100 \mathrm{MHz}$ and a PRF of $400 \mathrm{~Hz}$ over the test site of Oberpfaffenhofen, Germany. The flight altitude is $3200 \mathrm{~m}$, and the velocity of the platform is $91 \mathrm{~m} / \mathrm{s}$.

The data have been processed with a bandwidth half of the full PRF, leading to an effective PRF (PRF eff) of $200 \mathrm{~Hz}$, and a Hamming window $(\alpha=0.54)$ in both azimuth and range dimensions. Range registration has been carried out using the scaling properties of the extended chirp scaling algorithm (ECS) [8]. The original coherence and interferometric flattened phase appear in Figs. 1(a) and 2(a). The black stripes in the middle of Fig. 1(a) and in some other areas represent coherence losses due to residual motion errors. The Oberpfaffenhofen test site is almost flat, so that observed azimuth phase undulations in Fig. 2(a) are mainly due to residual motion errors.

To estimate the derivative of residual motion errors, spectral diversity has been configured with a separation between subapertures of $30 \mathrm{~Hz}$ ( 0.15 times the $\left.\mathrm{PRF}_{\text {eff }}\right)$, centered at $\pm 15 \mathrm{~Hz}$, and a bandwidth of also $30 \mathrm{~Hz}$. Using (2), residual motion errors have been estimated, and the suggested correction method has been applied to the data (50\% to each image). Figs. 1(b) and 2(b) show the corrected coherence and flattened phase, respectively. The improvement is evident; both registration errors and azimuth phase undulations disappeared almost completely. 


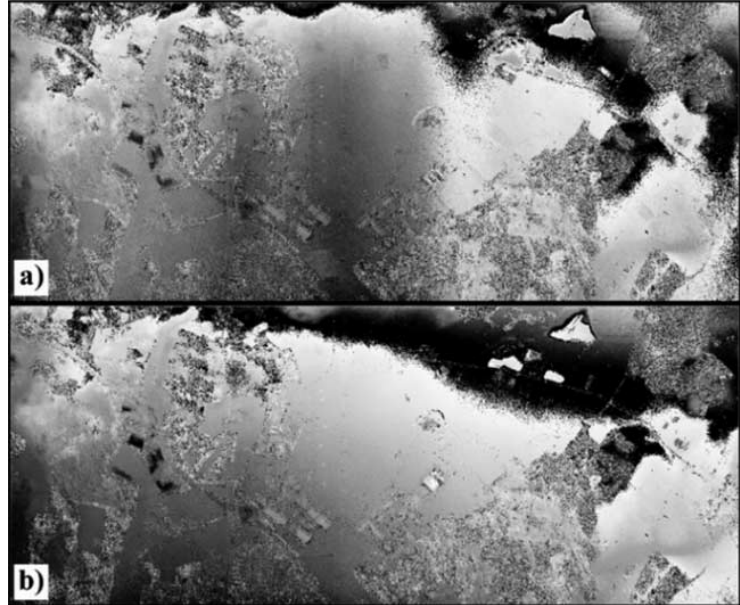

Fig. 2. Flattened phase (a) before and (b) after applying the proposed correction method.
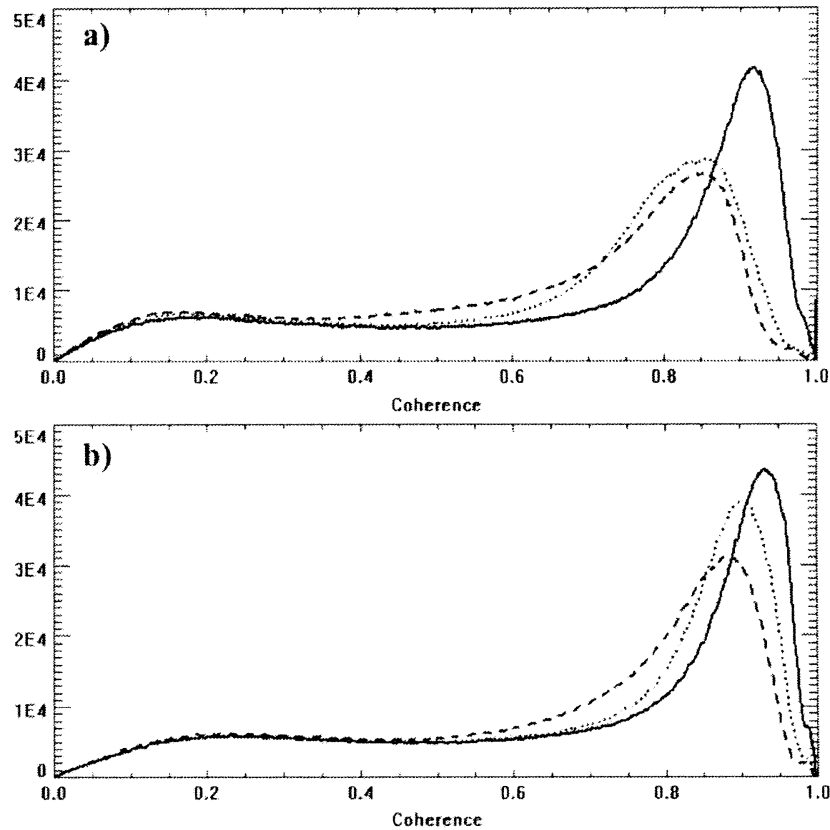

Fig. 3. Coherence histograms with data processed using a bandwidth (a) half of the PRF and (b) a quarter of the PRF. (Dashed line) Original coherence, (dotted line) corrected coherence using [3], and (solid line) corrected coherence using the proposed method.

Coherence histograms have been computed for the uncorrected interferogram, for the interferogram registered using [3] (with half of the $\mathrm{PRF}_{\text {eff }}$ for each subaperture, which is the optimum), and for the corrected interferogram using the proposed method, as shown in Fig. 3(a). The small improvement of coregistering using [3] is due to noncommon phase artifacts after azimuth focusing, as residual motion errors have not been corrected. Due to the bandwidth chosen for each subaperture, the estimated registration error using [3] is probably quite accurate, as the linear trend of residual motion errors is estimated. Note also that if one considers comparing the common procedure up to now (phase correction plus interpolation) with the proposed in this letter, then the dotted line in Fig. 3 corresponds to the former case. Effectively, applying a phase correction to one of the images does almost not affect the coherence, as a residual phase correction is usually slow variant. Therefore, in terms of coherence, the proposed algorithm performs significantly better than the common approach (phase correction, based either on Sections II-A or B, and interpolation).

The data have been also processed with a bandwidth of a quarter of the PRF. The histogram in Fig. 3(b) shows the result. In this case, the registration error of E-SAR data becomes almost linear along the synthetic aperture, so that the registration error estimated by [3] gives a very good result, although the proposed method is still the better approach. As the error is mainly linear along the synthetic aperture, almost no phase artifacts appear after azimuth focusing, leading to a high coherence after coregistration, contrary to what happened in the prior case.

\section{CONCLUSION}

An analysis of residual motion errors has been carried out, pointing out their two main consequences: azimuth phase undulations and azimuth registration errors. This allows to correct both in one step with a residual motion compensation, avoiding in this way the critical interpolation step commonly used to correct registration errors. Results with L-band data have been shown, proving the validity of the method.

The direct estimation of residual motion errors using spectral diversity [2] is recommended, as it does not make any assumption, leading to a more accurate result when used in conjunction with the proposed correction. Special care must be taken with the separation and bandwidth of subapertures, as they play an important role to be able to track fast variations. Furthermore, the algorithm can be easily made iterative for better detection of fast variations.

The proposed method can also be applied to single-pass systems, because errors in the measurement of the lever arms or rotation angles can lead to different motion errors in master and slave acquisitions, which can be detected and corrected using the proposed algorithms.

\section{ACKNOWLEDGMENT}

The authors would like to thank the German Aerospace Center (DLR) for supplying the E-SAR data. They would also like to thank the anonymous reviewers for their comments and suggestions.

\section{REFERENCES}

[1] A. Reigber, "Correction of residual motion errors in airborne SAR interferometry," Electron. Lett., vol. 37, no. 17, pp. 1083-1084, Aug. 2001.

[2] P. Prats and J. J. Mallorqui, "Estimation of azimuth phase undulations with multisquint processing in airborne interferometric SAR images," IEEE Trans. Geosci. Remote Sensing, vol. 41, pp. 1530-1533, June 2003.

[3] R. Scheiber and A. Moreira, "Coregistration of interferometric SAR images using spectral diversity," IEEE Trans. Geosci. Remote Sensing, vol. 38, pp. 2179-2191, Sept. 2000.

[4] R. Scheiber, "A three-step phase correction approach for airborne repeat-pass interferometric SAR data," in Proc. IGARSS, 2003.

[5] M. Bara, J. Monne, and A. Broquetas, "Navigation systems requirements for airborne interferometric SAR platforms," in Proc. IGARSS, 1999, pp. 2158-2160.

[6] M. Bara, R. Scheiber, A. Broquetas, and A. Moreira, "Interferometric SAR signal analysis in the presence of squint," IEEE Trans. Geosci. Remote Sensing, vol. 38, pp. 2164-2178, Sept. 2000.

[7] C. Cafforio, C. Pratti, and F. Rocca, "SAR data focusing using seismic migration techniques," IEEE Trans. Aerosp. Electron. Syst., vol. 27, pp. 194-207, Mar. 1991.

[8] A. Moreira, J. Mittermayer, and R. Scheiber, "Extended chirp scaling algorithm for air- and spaceborne SAR data processing in stripmap and scansar imaging modes," IEEE Trans. Gesoci. Remote Sensing, vol. 34, pp. 1123-1136, Sept. 1996. 